\title{
El futuro del presente. El Seminario Permanente de Multi, Inter y Transdisciplina en la Universidad Iberoamericana: una respuesta a los nuevos retos de la educación superior
}

The Future of the Present. The Permanent Seminar on Multi, Inter and Transdisciplinarity at the Universidad Iberoamericana: A Response to the New Challenges of Higher Education

\author{
Luis Javier Cuesta Hernández \\ Universidad Iberoamericana Ciudad de México, México \\ luis.cuesta@ibero.mx \\ Berenice Pardo Hernández \\ Universidad Iberoamericana Ciudad de México, México \\ marisela.pardo@ibero.mx
}

https://10.48102/didac.2021..78_JUL-DIC.74

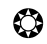

\section{RESUMEN}

Frente a la enorme complejidad de los problemas sociales actuales, las instituciones educativas tienen el deber moral y ético de plantearse formas de enseńanza-investigación que no se limiten al diálogo disciplinar, sino que diseñen maneras creativas de aportaciones epistemológicas transdisciplinares. Una vez abierto el camino para la reflexión respecto al futuro de la docencia y los retos a los que se enfrenta la pedagogía, el Seminario Permanente de Multi, Inter y Transdisciplina surge como una iniciativa de la Vicerrectoría Académica, encabezada por la maestra Sylvia Schmelkes, en la Universidad Iberoamericana Ciudad de México. Los resultados de este seminario ya se están poniendo a prueba para repensar los desafíos de desarrollo sustentable y las Preferencias Apostólicas Universales de la Compañía de Jesús desde distintas disciplinas con incidencia en el mundo real.

Palabras clave: Interdisciplina; multidisciplina; transdisciplina; pensamiento crítico; problemas sociales; aproximaciones a la investigación; resolución de problemas; sustentabilidad; complejidad. 


\begin{abstract}
Facing the enormous complexity of current social problems, educational institutions have a moral and ethical duty to consider forms of teaching/research not limited to disciplinary dialogue, but rather design creative ways of having transdisciplinary epistemological contributions. Once the path has been opened for reflection regarding the future of teaching and the challenges that pedagogy currently faces, the Permanent Multi / Inter / Transdisciplinary Seminar arises as an initiative of the Academic Provost, Sylvia Schmelkes, from the Universidad Iberoamericana, Mexico City. The results of this seminar are already being tested to rethink the challenges of sustainable development and the Universal Apostolic Preferences of the Society of Jesus, from different disciplines with incidence in the real world.
\end{abstract}

Keywords: Interdiscipline; Multidiscipline; Transdiscipline; Critical Thinking; Social Problems; Research Approaches; Problem Solving Focus; Sustainability; Complexity.

Fecha de recepción: 18/03/2021

Fecha de aceptación: 24/04/2021

\section{Introducción}

En los últimos años ha incrementado exponencialmente la gravedad de los problemas que el mundo enfrenta. Una muestra de la importancia de atender este fenómeno fue lo ocurrido el 25 de septiembre de 2015, cuando, a través de la formulación de los objetivos de desarrollo sustentable del milenio, y con el acuerdo de los principales líderes mundiales, se adoptaron un conjunto de proyectos globales para erradicar la pobreza y proteger el planeta.

Asimismo, la publicación de la encíclica papal Laudato si'y de las Preferencias Apostólicas Universales de la Compañía de Jesús coincidieron en el cuidado de la Casa Común al promover la conversión ecológica de la humanidad. Finalmente, y ante la actual pandemia producida por la COVID-19, surge con mayor fuerza la necesidad de pensar en el destino bioecológico del planeta y de las sociedades humanas.

La Universidad Iberoamericana (parte del Sistema Universitario Jesuita) no es ajena a esto y siempre ha demostrado una gran preocupación por relacionar los saberes impartidos con la solución real de problemas sociales complejos. Esta vocación puede rastrearse, entre líneas, tanto en la estructura departamental de la Universidad como en la creación de los primeros institutos de investigación, iniciativas que permitieron el abordaje de los problemas sociales a través de las aportaciones epistemológicas. Con esto se dieron los primeros pasos o se pusieron las bases hacia prácticas docentes y de investigación interdisciplinares.

En el año 2018, y como respuesta a la inquietud sobre la grave situación económica, social y ambiental del mundo, la Vicerrectoría Académica de la Universidad Iberoamericana impulsó la creación del Centro Transdisciplinar Universitario para la Sustentabilidad (Centrus).

Con esto, la institución se sumaba a muchos esfuerzos ya existentes en torno a los temas de desarrollo y medio ambiente. El Centrus concibe dimensiones del desarrollo relacionadas con las personas, la prosperidad, la paz y el planeta (Guevara Sanginés et al., 2019).

El proyecto más ambicioso hasta ahora de dicha unidad académica fue la creación de la licenciatura en Sustentabilidad. Con el objetivo de generar alternativas de solución a problemas de sustentabilidad (ambientales, sociales y económicos) — desde una perspectiva interdisciplinar y con un programa que se empezaba a definir como transdisciplinar- se pretendía que el estudiante estuviera involucrado en problemas reales, para fortalecer así el modelo de relación academia-sector privado-gobierno. También se buscaba que los académicos proyectaran su labor hacia el exterior, que tuvieran vínculos con los demás sectores de la sociedad y que produjeran investigación útil para su transformación.

A su vez, la creación y la puesta en marcha del doctorado en Estudios Críticos de Género fue otra 
de las iniciativas que implementó la Universidad Iberoamericana para continuar la reflexión en torno a problemas que cruzaban la vida diaria de los individuos en el mundo. Gracias a su carácter interdisciplinar y a la incorporación de la teoría crítica en su aparato epistémico, el programa ha integrado elementos de los departamentos de Arte, Ciencias Sociales y Políticas, Ciencias Religiosas, Comunicación, Derecho, Desarrollo Humano, Economía, Estudios Internacionales, Filosofía, Literatura, Historia y Psicología.

A través de las teorías y las metodologías de estudios críticos y de estudios de género, este programa se percibe, piensa, elige y actúa a favor de los derechos humanos y de la construcción de relaciones humanas y sociales inclusivas, igualitarias, libres, amorosas, justas y con perspectiva ética para conformar comunidades respetuosas de la dignidad humana y de los libres procesos personales de subjetivación.

De esta manera, tanto la licenciatura en Sustentabilidad como el doctorado en Estudios Críticos de Género constituían, hasta aquel momento, las iniciativas interdisciplinares más destacadas de la Universidad Iberoamericana.

\section{Primeros pasos y estado de la cuestión}

Abierto el camino para la reflexión respecto al futuro de la docencia y a los retos a los que se enfrenta la pedagogía, el Seminario Permanente de Multi, Inter y Transdisciplina surgió como una iniciativa de la Vicerrectoría Académica, encabezada por la maestra Sylvia Schmelkes, de la Universidad Iberoamericana Ciudad de México, a partir de conversaciones enmarcadas en el proceso de Planeación Estratégica desde 2018.

Fue así que, en la primavera de 2019, y bajo la coordinación de la División de Humanidades y Comunicación, dieron inicio las reuniones periódicas del seminario, con la idea de tener un punto de encuentro y reflexión entre diversas disciplinas y convertirse en el espacio ideal para continuar con el debate en torno a todos estos problemas.

En noviembre 2019, y con una afluencia superior en ocasiones a los 100 miembros de la comuni- dad universitaria, se acordó la realización de una semana intensiva de actividades que permitiera poner las bases teóricas, epistemológicas y pedagógicas para trabajar con futuros planes de estudio.

De manera colegiada, el Seminario Permanente eligió a dos especialistas en la materia — las doctoras Ulli Vilsmaier y Dena Fam-, quienes llevarían a cabo talleres-seminarios para un trabajo integral con la comunidad académica.

El objetivo principal era profundizar en los conceptos teóricos fundamentales de la multi, inter y transdisciplina. Mientras que los objetivos secundarios eran:

1. Construir conocimientos y habilidades para el desarrollo de nuevos planes de estudio transdisciplinares.

2. Reflexionar sobre nuevas formas organizacionales al interior de los departamentos y las divisiones interdepartamentales.

3. Resaltar como prioridad fundamental el análisis metodológico de los modelos transdisciplinarios de las instituciones a las que pertenecen Ulli Vilsmaier y Dena Fam.

4. Finalmente, y como parte de las actividades, se invitó al doctor Alexander Nemerov - Carl y Marilynn Thoma Professor en el Departamento de Artes y Humanidades de la Universidad de Stanford-, quien impartió conferencias magistrales y seminarios para fortalecer el aspecto transdisciplinario de la investigación, en el que es una de las máximas autoridades a nivel mundial.

Desde la concepción del seminario, consideramos fundamental tomar en cuenta las definiciones mínimas de "disciplina" y sus contrapartes de multi, inter y transdisciplina; así como sus desarrollos críticos durante los últimos años y las reflexiones organizacionales en torno a las prácticas en distintos campos del quehacer académico.

El concepto de "disciplina" se deriva del latín discere (que comparte su raíz con aprendizaje) que, a su vez, conduce a la doctrina. Sin embargo, en este

110 - El futuro del presente. El Seminario Permanente de Multi, Inter y Transdisciplina en la Universidad Iberoamericana... Luis Javier Cuesta Hernández y Berenice Pardo Hernández. DIDAC 78 (2021): 108-116 
enfoque etimológico, la doctrina sin conocimiento del mundo no tendría sentido, por lo que debe incorporarse a ella la "ética del discurso, en el cual, Habermas define el mundo del sistema y el mundo de la vida" (Henao Villa et al., 2017, p. 179). En este punto filosófico, comprendemos que la disciplina forma parte de un sistema social de conocimiento del mundo objetivo por parte del sujeto.

Con base en lo anterior, podríamos afirmar que la función de la disciplina es proporcionar un conocimiento sistematizado del mundo. No obstante, el conjunto de conocimientos que se despliegan de las distintas disciplinas y que se abocan a un solo campo de estudio se ha visto, a lo largo de la historia de la epistemología, desbordado.

Por este motivo, la hiperespecialización de una disciplina no lleva única y de manera exclusiva a las entrańas de la misma (al menos no al intentar resolver situaciones socialmente complejas), sino que, de manera inevitable, nos hace voltear hacia otras disciplinas para buscar respuestas a los problemas complejos, mismos que, por su dificultad y debido a las implicaciones de orden social, obligatoriamente nos ponen en la necesidad de dialogar más allá de sus fronteras.

El concepto de "multidisciplina" se ha manejado desde la década de 1970. En principio, se teorizó como "compuesto o hecho de varias franjas especializadas del conocimiento, en la búsqueda de un objetivo común" (Random House College Dictionary, 1975). Sin embargo, la literatura en torno a su definición se ha desarrollado y complejizado para su mejor comprensión a lo largo de los años.

Una de las autoridades mundiales que ha trabajado con el Seminario Permanente define con pertinencia y actualidad los términos correspondientes a la utilidad pedagógica de la multidisciplina: "la aproximación multidisciplinaria requiere que cada estudiante resuelva un problema en común, contribuyendo desde su propio espectro de experiencia” (Fam et al., 2018, p. 88).

Menos conservador y con un uso más extendido en la práctica académica, el concepto tradicional de la interdisciplina se define como "una combinación de varias disciplinas en la búsqueda de un objetivo, no necesariamente trabajado de forma integrada o coordinada" (International Rice Research Institute, 2005, p. 182, en Henao Villa et al., 2017). Efectivamente, la dinámica de la interdisciplina requiere que "los miembros de un equipo con investigadores procedentes de distintos ámbitos del conocimiento se comprometan en un nivel profundo de colaboración para alcanzar objetivos planteados en común" (Bernard-Bonnin et al., 1995, p. 183, en Henao Villa et al., 2017).

No obstante, la literatura más actualizada hace hincapié en que la interdisciplina requiere de una gran compenetración y disposición en el ámbito de la educación, pues esta modalidad de enseñanza y aprendizaje exige que el educador tenga conocimiento de distintas disciplinas y experiencia en la forma en que el conocimiento se puede socializar desde cada una de éstas. "El objetivo es la creación de nuevos bloques de conocimiento, precisamente a través de procesos de aprendizaje desde metodologías distintas, en constante diálogo" (Fam et al., 2018, p. 89).

Como podemos percibir casi de inmediato, uno de los mayores problemas de las discusiones respecto a la interdisciplinariedad tiene que ver con la comunicación entre pares de la misma y de otras disciplinas:

\footnotetext{
De acuerdo con Nicolescu (1997), este proceso debería llevar a la creación de una nueva disciplina o área de investigación científica. Sin embargo, McGregor (2004) argumentaba que, aunque los miembros del mismo equipo de investigación contribuyen en el proceso, todavía se hallan enraizados en sus propias disciplinas. (Schmalz et al., 2019, p. 389).
}

Finalmente, y casi como conclusión natural, la transdisciplina se debería enfocar en la agilidad con la que diferentes disciplinas, dentro y fuera del ámbito académico, se comunican y socializan el conocimiento para solucionar un problema. "El objetivo es usar el conocimiento creativamente, sin importar el bagaje disciplinario; aprender a través del proceso de la resolución de problemas" (Fam et al., 2018, p. 88). 
Otra de las colaboradoras internacionales del Seminario Permanente presentó una visión en la que "habría que entrecruzar y complementar las propuestas de entidades independientes, disciplinas académicas, culturas, comunidades y otros grupos sociales, todos en condiciones de crear una colaboración real, con resultados enfocados a un ejercicio de transdisciplina culturalmente sensible y crítico" (Freeth \& Vilsmaier, 2020, p. 57).

Sin embargo, resulta fundamental destacar que, si con las prácticas interdisciplinares ya es complicado "traducir las aspiraciones de colaboraciones interdisciplinarias productivas y significativas, en proyectos científicos exitosos" (Freeth \& Vilsmaier, 2020, p. 57), la interdisciplina implica, por naturaleza, un reto mucho mayor, tanto para los académicos como para los estudiantes y, sobre todo, para las instituciones educativas en búsqueda de un futuro sostenible.

Antes de seguir profundizando en los problemas inherentes a cada una de estas aproximaciones, no queremos dejar de mencionar una de las definiciones terminológicas más ilustrativas y, por qué no, más didácticas y divertidas de la multi, inter y transdisciplina:

Choi y Pak (2006) describieron las diferencias usando comida como analogía. En ese sentido, vincularon a la multidisciplinariedad con una ensalada (donde diferentes ingredientes se unen pero mantienen distinta identidad); interdisciplinariedad con un guisado como el estofado (donde los componentes están unidos para crear algo diferente, pero los ingredientes individuales todavía son identificables), y la transdisciplinariedad con un pastel (donde los ingredientes se combinan, pero el producto final es algo completamente nuevo y los primeros son, en su mayoría, indistinguibles) (Schmalz et al., 2019, p. 390).

La transdisciplina es la forma de investigación colaborativa a la que deberían aspirar programas que tengan como objetivo la incidencia social en una comunidad específica, para beneficio de sus habitantes y del medio ambiente. Como una forma de practicar la investigación que sintetiza el conocimiento de un amplio rango de disciplinas, la transdisciplina tiene el potencial de resolver problemas de la vida real porque crea oportunidades para la aplicación práctica en ámbitos específicos y de urgencia actual, como la sustentabilidad.

Aunque la transdisciplina no constituya en sí misma un término novedoso, sí se trata, posiblemente, del futuro de la investigación dentro y fuera de las aulas; como bien recuerda Dena Fam, esta práctica ya se le atribuía al filósofo de la educación Jean Piaget (Suiza) y a Erich Jantsch (Austria) (Fam et al., 2017), por lo que sus orígenes son rastreables en la historia de la pedagogía y sus aplicaciones. Aunque provenga de ese momento el lenguaje propio para describir las diferencias entre multi, inter y transdisciplina, nos toca a nosotros la noción de urgencia sobre cómo lograr un consenso en la resolución de problemas del mundo real. Hablemos ahora de nuestros intentos a ese respecto.

Últimos avances del Seminario Permanente de Multi, Inter y Transdisciplina

En febrero de 2020 se convocó a la primera reunión plenaria del Seminario Permanente de Multi, Inter y Transdisciplina en ese año. En este encuentro se tomó la decisión de conformar seis grupos de trabajo integrados por distintos académicos con el objetivo de discutir la vigencia y la validez de los temas-ejes-problemas que se habían abordado en momentos previos. Los temas se nombraron de la siguiente manera:

1. Estudios Territoriales

2. Sustentabilidad

3. Ciudad

4. Desigualdad

5. Estudios sobre Migración

6. Paz y Gobernanza

A pesar de la pandemia producida por la COVID-19, dedicándole tiempo personal, los integrantes del Seminario Permanente continuaron con los trabajos y mantuvieron reuniones periódicas cada quince días 
(el auxilio de la tecnología fue fundamental para no detener ninguna reunión y sacar adelante los compromisos acordados).

El primer producto de esos trabajos durante buena parte de 2020 puede definirse como seis documentos con propuestas de programas académicos coherentes con sus temas (y desarrollados de acuerdo con un problem solving focus).

Estos documentos fueron expuestos tanto en reuniones plenarias del seminario, como a los miembros de la Vicerrectoría y la Rectoría de la Universidad Iberoamericana, siempre con críticas muy positivas.

La metodología empleada en todos los casos incluyó discusiones epistémicas, intercambio interdisciplinar y la revisión de programas nacionales e internacionales comparables y preexistentes.

Tras este ejercicio, los grupos tomaron las siguientes decisiones:

1. El grupo de Estudios Territoriales detectó que esos programas no contemplaban visiones alternativas de territorio que se enfocaran en el desarrollo inclusivo y sostenible (valoración del patrimonio cultural, los productos de origen, la biodiversidad y la etnicidad). Tomando en cuenta que en México existen disparidades regionales que se manifiestan en el nivel de ingresos, la migración, el índice de pobreza, la falta de distribución de la riqueza, la educación, la salud, la infraestructura, los patrones de exclusión social y el deterioro del medio ambiente, entre otros, este grupo propuso un programa académico de maestría que responda a los retos particulares, con soluciones desde la sinergia de conocimientos generados por distintas disciplinas, produciendo tanto analistas territoriales que comprendan profundamente los territorios, desde la inclusión, el conocimiento del contexto local y con competencia para interpretar la realidad, como dinamizadores territoriales, capaces de identificar una realidad disonante y generar propuestas para modificarla. Esta visión implica buscar nuevos enfoques que promuevan un desarrollo inclu- sivo y sostenible, a partir de la valorización del patrimonio cultural, los productos de origen y la biodiversidad. Es notable el enfoque interdisciplinario en dicho programa, pero también su pretensión transdisciplinaria que se alcanzará en un futuro cercano.

2. El grupo de Sustentabilidad coincidió en que la humanidad se encuentra en una profunda crisis socioambiental, con múltiples manifestaciones de deterioro y agotamiento de la naturaleza, producto de una visión predatoria y antropocéntrica que ha propiciado la explotación ilimitada del entorno natural. Los miembros del grupo confirmaron que necesitan profesionistas que cuenten con compromiso, valores, conocimiento y capacidades para trabajar por la sustentabilidad desde todos los ámbitos y niveles de acción. Por lo tanto, la posibilidad de una maestría en Sustentabilidad, con una especialidad integrada, es más que necesaria. Este programa estaría dirigido a profesionistas egresados de diversas licenciaturas (biología, geografía, economía, derecho, ingenierías, relaciones internacionales o sociología) y que estén interesados en resolver, desde un enfoque territorial y una perspectiva inter y transdisciplinaria, problemas de la sociedad en su camino hacia la sustentabilidad.

3. El grupo de Ciudad analizó posibles temáticas para completar un futuro programa de licenciatura. Los miembros del grupo compartieron algunos temas para acotar el problema:

- Migración campo-ciudad

- Salud y espacio público

- Gestión cultural

- Conectividad

- Brecha digital y ciudades inteligentes

- Justicia ambiental y empoderamiento ciudadano

- Derecho a la ciudad

De esta manera, se pretendería crear un programa de licenciatura en Resiliencia Urbana y Cam- 
bio Social (con énfasis en salud y alimentación, brecha digital y soberanía tecnológica, espacio público y empoderamiento ciudadano, respuesta organizada a desastres naturales y sociales, entre otros) dirigida a todas las licenciaturas de la Universidad Iberoamericana.

4. El grupo de Desigualdad propuso la posibilidad de crear una licenciatura en Desigualdad. El grupo trata de definir el concepto poliédrico de desigualdad, con la aportación epistémica de cada uno de sus integrantes y desde sus disciplinas. De esa forma, se llegó a la conclusión de que el tema de la desigualdad en México es, sin duda, uno de los principales problemas a enfrentar:

- México está dentro del $25 \%$ de los países con mayores niveles de desigualdad en el mundo; más de 52 millones de personas viven en situación de pobreza, 74 de cada 100 mexicanos que nacen en los estratos más pobres no logran ascender de posición socioeconómica (CEEY) y $1 \%$ de la población en México concentra el $43 \%$ de la riqueza.

- La desigualdad se relaciona con, y se manifiesta en diferentes aspectos, como la discriminación, la violencia, la falta de oportunidades educativas, laborales, entre otras.

- Además, de acuerdo con la Organización de las Naciones Unidas para la Educación, la Ciencia y la Cultura (Unesco), entre las distintas dimensiones, configuraciones y contextos de la desigualdad, las cuales se entrelazan entre sí, podemos encontrar: la desigualdad económica, la desigualdad social, la desigualdad cultural, la desigualdad política, la desigualdad ambiental, la desigualdad espacial y la desigualdad de conocimiento.

5. El grupo de Estudios sobre Migración se dedicó a realizar una serie de propuestas de modificaciones y retroalimentaciones para una incorporación efectiva del concepto transdisciplinar en la maestría en Estudios sobre Migración (MEM) y el diplomado sobre Migración e Intervención Social, ambos pertenecientes al Departamento de Estudios Internacionales. Las propuestas generadas señalan que dicha maestría debería abordar temáticas como salud, crimen organizado, seguridad ciudadana y Guardia Nacional, construcción de la memoria, migración en relación con el cambio climático, para lo cual debería plantearse un carácter interdepartamental y un abordaje teórico interdisciplinar.

6. El grupo de Paz y Gobernanza planteó una solución similar, con una intervención en el programa de Especialidad sobre Paz y Gobernanza, que apenas se encontraba en las últimas fases de su implementación y apertura. Esa intervención se articularía con propuestas para: a) considerar el estudio integral del fenómeno de la corrupción y su incidencia en el detrimento de la calidad de vida por la degradación de los recursos naturales; b) tener presente la transformación del concepto de "paz y gobernanza" a través de la historia, y c) el diseño, desarrollo y evaluación de programas educativos enfocados a la promoción de la paz a partir de la realización y dominio de metodologías pedagógicas.

\section{Reflexión final}

El año 2020 significó un punto de inflexión histórico en el mundo. Específicamente en el ámbito de la academia y la educación, implicó un momento crítico para la capacidad de nuestra especie a la adaptación de una nueva era. Es momento de poner a prueba nuestros parámetros éticos, científicos y la noción de comunidad para sobrellevar retos a mediano y largo plazo.

Así, "el incremento de recursos digitales ha llevado a percepciones fluctuantes respecto al estilo de enseñar y aprender en las universidades, con el sentimiento de que éstas deberían proveer más contenido basado en clases" (Fam et al., 2018, p. 86), y esto nos lleva a cuestionar cuál es el sentido del aula en el ámbito de la pandemia (y pospandemia), así como el de la utilidad real de las disciplinas aisladas en su propio campo de investigación. 
La Universidad Iberoamericana está ilusionada con los resultados de este primer Seminario Permanente, el cual nos ha llevado a observar el crecimiento y la puesta en marcha de un programa de gran envergadura como Centrus, cuya incidencia en el mundo actual se hace cada vez más evidente como un ejemplo exitoso de transdisciplinariedad, pues "la sustentabilidad es muchas cosas a la vez y navega en territorios interesantes - es una meta, un ideal, una sombrilla y una subdisciplina de múltiples disciplinas. Es, también, inherentemente, transdisciplinaria” (Stock \& Burton, 2011, p. 1092).
Ojalá pueda ser ése el sendero que se promueva desde la Universidad Iberoamericana con el objetivo de fijar metas claras para la resolución de problemas con la conciencia y la sensibilidad cultural requeridas, pues la competitividad y la eficiencia en el mundo moderno de las ciencias y las humanidades requieren de una visión integral de las múltiples y complejas realidades que vivimos y viviremos en este vertiginoso siglo XXI. Estaremos listos para apoyar en ese sendero en cuanto sea necesario. ID]

\section{REFERENCIAS}

Fam, D., Palmer, J., Riedy, C. \& Mitchell, C. (2017). Transdisciplinary Research and Practice for Sustainability Outcomes. Nueva York: Routledge.

Fam, D., Leimbach, T., Scott, K., Hitchens, L. \& Callen, M. (2018). Meta-considerations for Planning, Introducing and Standardising Inter and Transdisciplinary Learning in Higher Degree Institutions: The Art of Collaborative Research and Collective Learning. En D. Fam, L. Neuhauser \& P. Gibbs (Eds.), Transdisciplinary Theory, Practice and Education (pp. 85-102). Suiza: Springer. Recuperado de http://dx.doi.org/10.1007/978-3-319-93743-4_7

Freeth, R. \& Vilsmaier, U. (2020). Researching Collaborative Interdisciplinary Teams: Practices and Principles for Navigating Researcher Positionality. Science \& Technology Studies, 33(3), 57-72.

Guevara Sanginés, A., Lara Pulido, J. A. \& Riojas Rodríguez, J. (2019). Investigación inter y transdisciplinaria en el cuidado de la Casa común: la propuesta del Centro Transdisciplinario Universitario para la Sustentabilidad (Centrus).
En G. Fernández Anaya, A. González Jácome, G. Prado Garduño \& M. C. Torales Pacheco (Eds.), La Universidad Iberoamericana generadora de conocimiento. Un caleidoscopio. Ciudad de México: Universidad Iberoamericana.

Henao Villa, C. F., García Arango, D. A., Aguirre Mesa, E. D., González García, A., Bracho Aconcha, R., Solórzano Movilla, J. G. \& Arboleda López, A. P. (2017). Multidisciplinariedad, interdisciplinariedad y transdisciplinariedad en la formación para la investigación en ingeniería. Revista Lasallista de Investigación, 14(1), 179-197.

Schmalz, D. L., Janke, M. C. \& Payne, L. L. (2019). Multi-, Inter-, and Transdisciplinary Research: Leisure Studies Past, Present, and Future. Journal of Leisure Research, 50(5), 389393. Recuperado de https://doi.org/10.1080/00222216.2 019.1647751

Stock, P. \& Burton, R. J. F. (2011). Defining Terms for Integrated (Multi-Inter-Trans-Disciplinary) Sustainability Research. Sustainability, 3(8), 1090-1113. Recuperado de https://doi.org/10.3390/su3081090

BiBLIOGRAFÍA RECOMENDADA

Aspeé Chacón, J. (2013). Disciplina, Interdisciplina, Transdisciplina: implicancias para el Trabajo Social. Recuperado de http:// www.trabajadoressociales.cl/provinstgo/articulo89.pdf

Bolio Paoli, F. J. (2019). Multi, inter y transdisciplinariedad. Recuperado de http://www.scielo.org.mx/scielo.php?script= sci_arttext\&pid=S2007-43872019000100347

Gamez, M. J. (2019). Objetivos y metas de desarrollo sostenible. Recuperado de https://www.un.org/sustainabledevelopment/ es/objetivos-de-desarrollo-sostenible/

Libro blanco sobre el desarrollo de programas interdisciplinares en la Universidad Iberoamericana. Hacia la resolución de problemas a través de la interdisciplina. Nuevas propuestas. Multil Inter/Transdisciplina. Debates y propuestas en la Universidad Iberoamericana. Manuscrito.

Lobos, N. (2016). Disciplinas, interdisciplina y transdisciplina. Lo cientifico de las ciencias sociales: entre los universales y la producción de lo concreto. Recuperado de https://reposito-
riosdigitales.mincyt.gob.ar/vufind/Record/BDUNCU_7ac1fdf36797249989e9bce6b4ec1819

Merçon, J., Ayala-Orozco, B. \& Rosell, J. A. (Coords.). (2018). Experiencias de colaboración transdisciplinaria para la sustentabilidad. México: CopIt-arXives. Publishing Open Access with an Open Mind.

Ruiz Gutiérrez, R. \&Martínez González, A. (2020). Multidisciplina e Interdisciplina en el posgrado de la UNAM. Recuperado de https://www.ceiich.unam.mx/Interdisciplina/posgrado.html

Santo Padre Francisco. (2015). Carta encíclica LAUDATO SI' sobre el cuidado de la casa común. Recuperado de http://www.vatican.va/content/francesco/es/encyclicals/documents/papa-francesco_20150524_enciclica-laudato-si.html

Sosa, A. (2019). Preferencias Apostólicas Universales. Cuidar de la Casa Común. Recuperado de https://www.jesuits.global/ es/uap/introduccion/ 
Sotolongo Codina, P. L. \& Delgado Díaz, C. J. (2006). Capítulo IV. La complejidad y el diálogo transdisciplinario de saberes. En La revolución contemporánea del saber y la complejidad social. Hacia unas ciencias sociales de nuevo tipo. Buenos Aires, Argentina: Clacso.
United Nations. Objetivos de desarrollo sostenible. Recuperado de https://www.un.org/sustainabledevelopment/es/objetivosde-desarrollo-sostenible/

SEMBLANZAS

Luis Javier Cuesta Hernández. Actual director de la División de Humanidades y Comunicación de la Universidad Iberoamericana Ciudad de México. También ha estado al frente del Departamento de Arte de la misma institución. Ha sido profesor invitado en diversas universidades de América Latina y España (Pontificia Universidad Católica de Chile, Universidad Autónoma de Madrid, Université Lumière Lyon 2). Doctorado cum laude en Historia del Arte por la Universidad de Salamanca, con la tesis El arquitecto Claudio de Arciniega en el virreinato de Nueva España. Vida y obra. Ha sido becario

C. B. Smith en el Teresa Lozano Long Institute of Latin American Studies de la Universidad de Texas y becario posdoctoral en el Instituto de Investigaciones Estéticas de la Universidad Nacional Autónoma de México (UNAM). Ha sido curador y asesor científico, entre otras, de la exposición

Michelangelo Buonarroti en el Museo del Palacio de Bellas Artes. Es autor de varios libros individuales, así como de numerosos capítulos de libros y artículos en ediciones colectivas y revistas científicas. También ha impartido conferencias internacionales en México, América y Europa. Actualmente es miembro del Sistema Nacional de Investigadores (SNI) del Consejo Nacional de Ciencia y Tecnología (Conacyt). Sus últimos libros: Ut architectura poesis. Relaciones entre arquitectura y literatura en la Nueva España durante el siglo XVII, Universidad Iberoamericana, 2013 y Trazos en la historia: arte español en México (coord.), Editorial El Viso, 2017.

Berenice Pardo Hernández. Licenciada en Historia del Arte por la Universidad Iberoamericana Ciudad de México, y maestra en Filosofía Política por la Universidad Pompeu Fabra de Barcelona. Ha sido asesora editorial en Fomento Cultural Banamex, A. C., agregada cultural en el Consulado de México en Barcelona, donde coordinó el Centenario de Octavio Paz en Cataluña, y directora del Museo Vizcaínas. Es autora del libro Mineral de la Luz. La obra fotográfica de John Horgan Jr. en México y coautora de De vicios y virtudes, de hechizos y conspiraciones están hechos los hombres. La Inquisición en Nueva España, publicado por la Facultad de Medicina de la unam. Ha coordinado eventos internacionales como gestora cultural y ha sido curadora de exposiciones en México y España, actividades que realiza de manera independiente. Actualmente está estudiando el doctorado en 17, Instituto de Estudios Críticos.

\footnotetext{
${ }^{1}$ Véase <https://sdgs.un.org/es/goals>. El pacto por la sustentabilidad ha sido refrendado por 193 países al firmar en 2015, en el seno de la Organización de las Naciones Unidas (ONU), el acuerdo sobre la Agenda 2030 para el Desarrollo Sostenible.

${ }^{2}$ Véase <http://www.vatican.va/content/francesco/es/encyclicals/documents/papa-francesco_20150524_enciclica-laudato-si.html>.

${ }^{3}$ Véase $<$ https://www.jesuits.global/uap/introduction/>.

${ }^{4}$ Los inicios fueron modestos. El seminario se reunía quincenalmente en la pequeña sala de juntas de las Divisiones Académicas y congregaba entre 10 y 15 académicos y directivos de la Universidad. La mayoría de los integrantes de ese núcleo inicial continúa los trabajos en el grupo expandido actual. ${ }^{5}$ Institute of Sustainable Futures, Sidney University (Australia); y Universidad Leuphana de Lüneburg (Alemania), respectivamente.

${ }^{6} \mathrm{El}$ profundo interés mostrado por los asistentes a estas presentaciones resultó muy satisfactorio, pero no por ello menos inquietante. Quienes participaron de manera interactiva mostraron su preocupación por la escasez de vías para trascender los marcos disciplinares, con el objetivo de incorporar en sus investigaciones marcos epistemológicos que sólo pueden ser alcanzados desde los avances transdisciplinares contemporáneos.

${ }^{7}$ Las cursivas son nuestras.

${ }^{8}$ Las cursivas son nuestras.

${ }^{9}$ Para aquel momento, el Seminario Permanente se encontraba integrado por varias decenas de académicos, investigadores y directivos universitarios. Ya se contaba, por lo tanto, con una masa crítica superior a $10 \%$ de la nómina de la Universidad Iberoamericana. En nuestra opinión, ese concepto de "masa crítica" se ha revelado fundamental en el avance del trabajo de estos grupos.

${ }^{10}$ Por una feliz coincidencia, ese programa académico se encontraba en medio de un proceso de desarrollo curricular. Se decidió aprovechar la convergencia de ambos procesos para convertirlo en un programa completamente transdisciplinar.

${ }^{11}$ Misma anotación que la anterior, con la salvedad de que este programa era de nueva creación, ya se encontraba en los procesos curriculares correspondientes, pero se aprovechó esta coyuntura para revisar nuevamente su propuesta.
}

116 - El futuro del presente. El Seminario Permanente de Multi, Inter y Transdisciplina en la Universidad Iberoamericana... Luis Javier Cuesta Hernández y Berenice Pardo Hernández. DIDAC 78 (2021): 108-116 